\title{
COVID-19 Testing in Young Individuals and Pandemics Monitoring: Low Susceptibility to the Infection and Lack of Positive Results
}

\author{
Watanabe ASA, Dias VC, Machado ABF, Diniz CG \\ and da Silva VL* \\ Department of Parasitology, Microbiology and \\ Immunology, Institute of Biological Sciences, Federal \\ University of J uiz de Fora, J uiz de Fora, MG, Brazil \\ *Correspondling author: Vânia Lúcia da Silva, \\ Department of Parasitology, Microbiology and \\ Immunology, Institute of Biological Sciences, Federal \\ University of J uiz de Fora. Rua J ose Lourenço Kelmer \\ s/ n, Bairro São Pedro, J uiz de Fora, MG, Brazil
}

Received: May 10, 2021; Accepted: J une 23, 2021;

Published: J une 30, 2021

\section{Letter to the Editor}

Severe Acute Respiratory Syndrome Coronavirus 2 (SARSCoV-2), a novel betacoronavirus, is the etiological agent of coronavirus disease 2019 (COVID-19), a global health threat. The rapid spread of COVID-19 has resulted in an urgent requirement for rapid diagnosis and effective therapeutic strategies against SARSCoV-2 [1]. Besides symptomatic patients, recent studies indicate that people who are infected but do not presented symptoms also play a role in the spread of COVID-19. Spread happens when an infected person coughs, sneezes, or talks, and aerosol/droplets from their mouth or nose are launched into the air and reaches the mucous membranes of people nearby [2].

Although some point-of-test assay and Enzyme Linked Immuno Sorbent Assay (ELISA) based protocols are available for COVID-19 diagnosis, the reverse transcriptase real-time quantitative polymerase chain reaction (RT-qPCR) remains as gold standard diagnostic tool and is supposed to present high sensitivity and reliability of viral genome in clinical specimens [3]. Healthcare Workers (HCW) are at high risk of SARS-CoV-2 infection and epidemiological features of its transmission into household are limited. Several families, mainly from HCW, are temporarily separated due to isolation and quarantine, and the risks associated with these professions and the dynamics of household transmission must be evaluated [4]. These questions are still more notorious in low income and developing countries, such as Brazil, once public health policies anti-COVID-19 is extremely fragile.

During COVID-19 pandemics, a 39-year-old Brazilian woman, intensive care physician at tertiary hospital in our region, in the third trimester of pregnancy, experiencing hyposmia and ageusia (temporary loss of smell and taste respectively), presenting cough, sneeze and nasal congestion, was diagnosed with COVID-19 by RT- qPCR. Other signs and symptoms such as fever, sore throat, dyspnea, oxygen saturation $<95 \%$, vomiting, respiratory distress or diarrhea was not observed. As an intensive care physician, she was immunized against Influenza virus (A and B) by vaccination early in the beginning of 2020 and was working in the frontline in a Brazilian tertiary hospital, where several patients tested positive for COVID-19 at the same time. Further, her husband (39 years old) and her son (13 years old) were also screened for COVID-19, by RT-qPCR, according to the disease transmission containment protocol. The husband tested also positive for COVID-19 while her son presented negative result for SARS- CoV-2 from the combined oropharyngeal and nasal swab. The husband reported sore throat, sneezing and headache. The son presented cough, nasal congestion and coryza. Until the moment of COVID-19 test, the husband and the child did not receive the 2020 Influenza virus ( $A$ and $B$ ) vaccine.

Frontline HCWs could be at high risk of infection due to the close contact with patients and reports have described COVID-19 cases in HCWs since early in the outbreak [5]. Moreover, HCWs with infection could cause secondary transmission among patients, family members, and the community. Therefore, it is important to investigate the infection risk of HCWs and the clinical characteristics of affected cases [6]. Risk factors for development of COVID-19 in exposed HCWs include suboptimal handwashing, improper use of personal protective equipment (PPE), longer long workday, and assignment to a high-risk department [7]. Preventing HCW infections is important for reducing morbidity and potential mortality, maintaining health system capacity, and reducing secondary transmission [8]. Especially in Brazil, which is currently considered the epicenter of the pandemics in South America and the entire world, still in the ascending curve of infection, the lack of strong containment policies may play important role in the exposure of HCWs and their households to the disease, as not only COVID-19 infected patients are the main infection sources to HCW [9]. In this context, it also should be important to state the asymptomatic cases, which may play a critical role in the transmission process. Respiratory droplets and contact are the main transmission routes. Close contact with asymptomatic and pre-symptomatic patients with silent infection are the important routes of COVID-19 transmission from HCW towards household [4]. The intensive care physician related is a frontline HCW, in a tertiary hospital, which had at the same time several confirmed cases of COVID-19. The data may strongly suggest the possibility of occupational SARS-CoV-2 infection and further COVID-19 transmission into household and even close contact community with exception of her 13-years- old son.

Although young individuals such as children and teenagers are likely to be susceptible to coronavirus infection if compared to adults, it has been observed that these patients consistently represent only 1-2 $\%$ of confirmed cases worldwide. In general, young patients such as children and teenagers tend to undergo a less severe disease than adults when they are infected with SARS-CoV-2 [10]. When not
J Fam Med - Volume 8 Issue 5 - 2021

ISSN : 2380-0658 | www.austinpublishinggroup.com

da Silva et al. (@) All rights are reserved
Citation: Watanabe ASA, Dias VC, Machado ABF, Diniz CG and da Silva VL. COVID-19 Testing in Young Individuals and Pandemics Monitoring: Low Susceptibility to the Infection and Lack of Positive Results. J Fam Med. 2021; 8(5): 1260. 
asymptomatic, they may present fever, dry cough, and fatigue, or few upper respiratory symptoms including nasal congestion and running nose. Young patients tend to recover within 1-2 weeks after disease onset; however, a few may progress to lower respiratory infections [11].

Although the reasons for the relative resistance of young individuals to some infectious diseases remain obscure, with regards to the COVID-19, it is suggested some reasons which include a more active innate immune response, healthier respiratory tracts related to the less exposure to tissue irritants such as cigarette smoke and air pollution if compared to adults, and fewer underlying disorders. A more vigorous immune response in adults may also explain a detrimental immune response associated with acute respiratory distress syndrome [12]. The clinical presentation observed in the father (mainly headache) may indicate a systemic symptom, unlike the son who presented symptoms of upper respiratory tract infection (cough, nasal congestion and coryza).

By the other hand, differences in the distribution, maturation, and functioning of viral receptors is frequently reported as a possible reason of the age-related difference in incidence of COVID-19. The SARS-CoV-2 and Human Coronavirus-NL63 (HCoV-NL63) all use the Angiotensin-Converting Enzyme-2 (ACE2) as the cell receptor in humans [13]. Recent findings have started to discuss the role of the endothelium in the outcome of infection, with the implication that young people may have a milder symptomatic infection when compared to adults. Increasing evidence points to a possible difference between adults and children whether it is the condition of their blood vessels. The hypothesis is that the endothelium of children would be more preserved when compared to adults, which could result in less inflammatory problems and less coagulation problems, two problems associated with unfavorable outcomes such as severe infection or death [14].

Another hypothesis to be considered is the viral load in children. Some authors describe that children may have a lower SARS-CoV-2 viral load when compared to adults, which could influence the diagnosis of COVID-19 [15].

\section{Final Considerations}

The data presented highlights the importance of HCW within the epidemiological chain as facilitators of the viral spread, such as the coronavirus, into household, family members and throughout the community. Quick and assertive diagnosis measures in the HCW infected with SARS-CoV-2, as well as the implementation of measures to contain the spreading of the virus are essential to control this pandemic. The observations may also sustain that young individuals may be less susceptible to COVID-19 and might interfere in the diagnostic method. According to the sanitary authorities worldwide, the supposed resistance to SARS-COv-2 infection and the COVID-19 negative testing in children has been though as pandemics monitoring subsidizing flexibilization social distancing polices. It is important further prospective studies in the COVID-19 clinical-microbiological characteristics and epidemiology in pediatric patients to elucidate underlying mechanisms which may help to manage COVID-19 in adults and discuss guidelines for RT-qPCR based SARS-CoV- 2 screening in these individuals.

\section{Acknowledgement}

This work was supported by Fundação de Amparo a Pesquisa do Estado de Minas Gerais (FAPEMIG), Conselho Nacional de Desenvolvimento Científico e Tecnológico (CNPq) and Coordenação de Aperfeiçoamento de Pessoal de Nível Superior (CAPES).

\section{Compliance with Ethical Standards}

Human Rights: This study was approved by the Ethics Committee of the Federal University of Juiz de Fora (protocol \#4.057.992), with a waiver of informed consent.

\section{Authors' Contribution}

ASAW: work's design, interpretation, and writing.

VCD: data collection, analysis, interpretation, and writing ABFM: analysis, interpretation, and writing.

CGD: manuscript writing, revision VLS: manuscript writing, revision.

\section{References}

1. Guan WJ, Ni ZY, Hu Y, Liang WH, Ou CQ, et al. Clinical characteristics of coronavirus disease 2019 in China. N Engl J Med. 2020; 382: 1708-1720.

2. Li Q, Guan X, Wu P, Wang X, Zhou L, et al. Early transmission dynamics in Wuhan, China, of novel coronavirus-infected pneumonia. N Engl J Med. 2020a; 382: 1199-1207.

3. Alcoba-Florez J, González-Montelongo R, Íñigo-Campos A, Artola DGM, GilCampesino $\mathrm{H}$, et al. Fast SARS-CoV-2 detection by RT-qPCR in preheated nasopharyngeal swab samples. Int J Infect Dis. 2020; 97: 66-68.

4. Li W, Zhang B, Lu J, Liu S, Chang Z, et al. The characteristics of household transmission of COVID-19. Clin Infect Dis. 2020b; 17: 450.

5. Wang D, Hu B, Hu C, Zhu F, Liu X, et al. Clinical characteristics of 138 hospitalized patients with 2019 novel coronavirus-infected pneumonia in Wuhan, China. JAMA. 2020; 323: 1061-1069.

6. Lai X, Wang M, Qin C, Tan L, Ran L, et al. Coronavirus Disease 2019 (COVID-2019) infection among health care workers and implications for prevention measures in tertiary hospital in Wuhan, China. JAMA. 2020; 3.

7. Chou R, Dana T, Buckley DI, Selph S, Fu R, et al. Epidemiology of and risk factors for Coronavirus infection in health care workers: a living rapid review. Ann Intern Med. 2020.

8. Adams JG, Walls RM. Supporting the health care workforce during the COVID-19 global epidemic. JAMA. 2020; 23: 1439-1440.

9. The Lancet. COVID-19 in Brazil: "So what?" Lancet. 2020; 39: 1461.

10. CDC COVID-19 Response Team. Coronavirus Disease 2019 in Children United States MMWR Morb Mortal Wkly Rep. 2020; 69: 422-426.

11. Chan JF, Yuan S, Kok KH, To K, Chu H, et al. A familial cluster of pneumonia associated with the 2019 novel coronavirus indicating person to-person transmission: a study of a family cluster. Lancet. 2020.

12. Lee PI, Hu YL, Chen PY, Huang YC, Hsueh PR. Are children less susceptible to COVID-19? J. Microbiol. Immunol. Infect. 2020; 53: 371-372.

13. Hofmann H, Pyrc K, van der Hoek L, Geier M, Berkhout B, et al. Human coronavirus NL63 employs the severe acute respiratory syndrome coronavirus receptor for cellularentry. Proc Natl Acad Sci. 2005; 102: 7988e93.

14. Varga Z, Flammer AJ, Steiger P, Haberecker M, Andermatt R, et al. Endothelial cell infection and endotheliitis in COVID-19. Lancet. 2020; 395: 1417-1418.

15. Liu Y, Yan LM, Wan L, Xiang TX, Le A, et al. Viral dynamics in mild and severe cases of COVID-19. Lancet Infect. Dis. 2020; 20: 656-657. 